\title{
Digital Smile Design
}

\author{
Ammar Kayssoun* \\ Department of Prosthodontics, Faculty of Dentistry, Near East University, Lefkosa, Turkey
}

Corresponding author: Ammar Kayssoun, Department of Prosthodontics, Faculty of Dentistry, Near East University, Lefkosa, 99138 Mersin 10, Turkey

\begin{tabular}{|c|c|}
\hline ARTICLE INFO & ABSTRACT \\
\hline Received: 慧 October 21, 2020 & Citation: Ammar Kayssoun. Digital Smile Design. Biomed J Sci \& Tech Res 31(3)-2020. \\
\hline Published: 幽October 27, 2020 & BJSTR. MS.ID.005099. \\
\hline
\end{tabular}

\section{Esthetic Digital Smile Design Introduction and View}

The importance given to a beautiful smile is not new. The search for beauty can be traced to the earliest civilizations; both the Phoenicians (app 800 BC) and Etruscans (app 900 BC) carefully carved animal tusks to simulate the shape, form and hue of natural teeth [1]. Dentists who are experienced with cosmetic treatment know that subtle variations in the shapes of the teeth can mean the difference between successful treatment and hours of reshaping, contouring, shortening, or worst of all, restarting a case. Orthodontic treatment is performed on a significant population of patients who are not satisfied with what nature has given them in their smiles. People spend significant amounts of money with plastic surgeons and dermatologists to look different than what was their natural appearance. Do we as dentists always want to give patients a smile that mirrors what is often found in nature, such as crowded, overlapped, and twisted teeth, malocclusions or diastemas [2].

To minimize extended or failed delivery visits with removable or fixed prosthetics because of patient dissatisfaction with the smile design, several smile guides have been developed over the years. These guides have attempted to serve as a communication tool to help the doctor understand what the patient wants, and for the doctor to communicate those desires to the laboratory. Nowadays, Computer design software will become the main communication technology between dentists and ceramists and a useful tool for showing patients the possibilities for enhancing their smiles. There are new, step-by-step techniques for incorporating digital technology into the smile design process that can be accomplished in approximately three to four minutes [3].

Aesthetic digital smile design DSD used to assist and improve diagnosis, communication, and predictability of treatment through an esthetic analysis of the assembly: Face, smile, periodontal tissue, and teeth. And the esthetics of the smile is related to the color, shape, texture, dental alignment, gingival contour, and the relationship of these with the face [4].

\section{DSD Method and Protocol}

DSD should first be an instrument to improve communication with the patient by showing the patient detailed images. On the monitor, the before and after photographs allow an index of predictability and point of comparison with the patient himself or herself. A milestone is the innovation of aesthetic clinical planning in aesthetic dentistry and prosthetic dentistry relating to dental technical analysis and planning, which among other things, can be integrated into diagnosis and planning for plastic and maxillofacial surgery [5]. The protocol first requires the acquisition of full-frame digital images as follows: full face with a wide smile, full face at rest, and retracted view of the full maxillary arch with teeth apart; and videos of the patient [4]. Video especially is capable of capturing the dynamic phases of the smile linked to its physiology (mimicry, phonetics, relationship between the teeth and lips).

Importing this vital data into the digital clinical file of the patient is complementary to the anamnesis because it is an integral part of the intra- and extraoral objective examination, and will subsequently be the subject of aesthetic analysis according to the main guiding principles. Therefore, we could define this as the third part of the methodology, which we will call analytical processing, during which the aesthetic composition of the smile, the determining morphological features of the face and smile, including the fundamental points of reference to be obtained from software such as face makers, will be mapped and processed. The next phase 
in digital data processing is virtual planning by means of digital image editing: Wax-up, digital and analogue diagnosis, mock- up, and provisional and definitive restorations. The digital methodology used for photograph and image editing is very reliable, especially in communicating through images the ongoing clinical case to dental laboratories concerning functional and morphological adjustments, which is made even easier if accompanied by explanations and verbal comments [5].

\section{Acquisition and Import of Digital Images}

As stated earlier, the first phase of DSD entails the acquisition and import of photographs of the patient. If possible, these photographs should be taken with a digital SLR camera with semi- professional features and with a good illumination system. That in the analytical phase the photograph is a clinical and aesthetic diagnostic element that will form part of the patient's clinical history, which can be consulted by other specialists to establish an interdisciplinary vision. In view of this, the dentist/photographer must capture the photographs with the patient's head in a position that can be replicated in the future to verify topography in relation to smile design (Figure 1). Most reliable position in which to photograph the patient's face is that relative to the aesthetic plan, that is the plane perpendicular (frontal) to the plane that runs at the center of the angle formed between the Frankfort horizontal plane and Camper's plane.

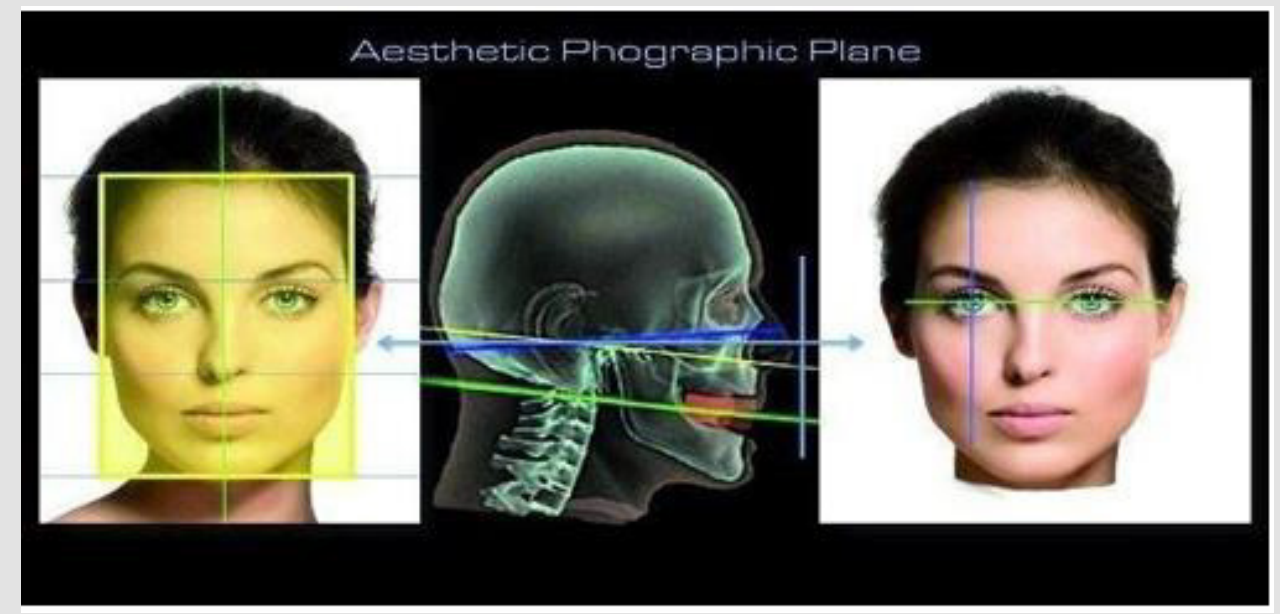

Figure 1: Photographs and aesthetic plane.

DSD imports the measurements of the photographed subject standardized and configured to the scale of values expressed in pixels, the ordinary unit of measurement of a digital photograph. In order to do this, it is possible to use technical drawing tools, such as set squares and rulers (made of metal if possible and thus easily cleaned and capable of being sterilized, or other similar material).
DSD built a measuring tool called Face Analogic Transfer Support (Figure 2), which consists of a ruler with graduated millimeter and centimeter scales, which the patient can wear like a pair of glasses. Furthermore, for new photographs for the fabrication of mock-ups and PMMA models etc. it is useful to use a device such as a craniostat fixed to the headrest, which is integrated into our dental chair.

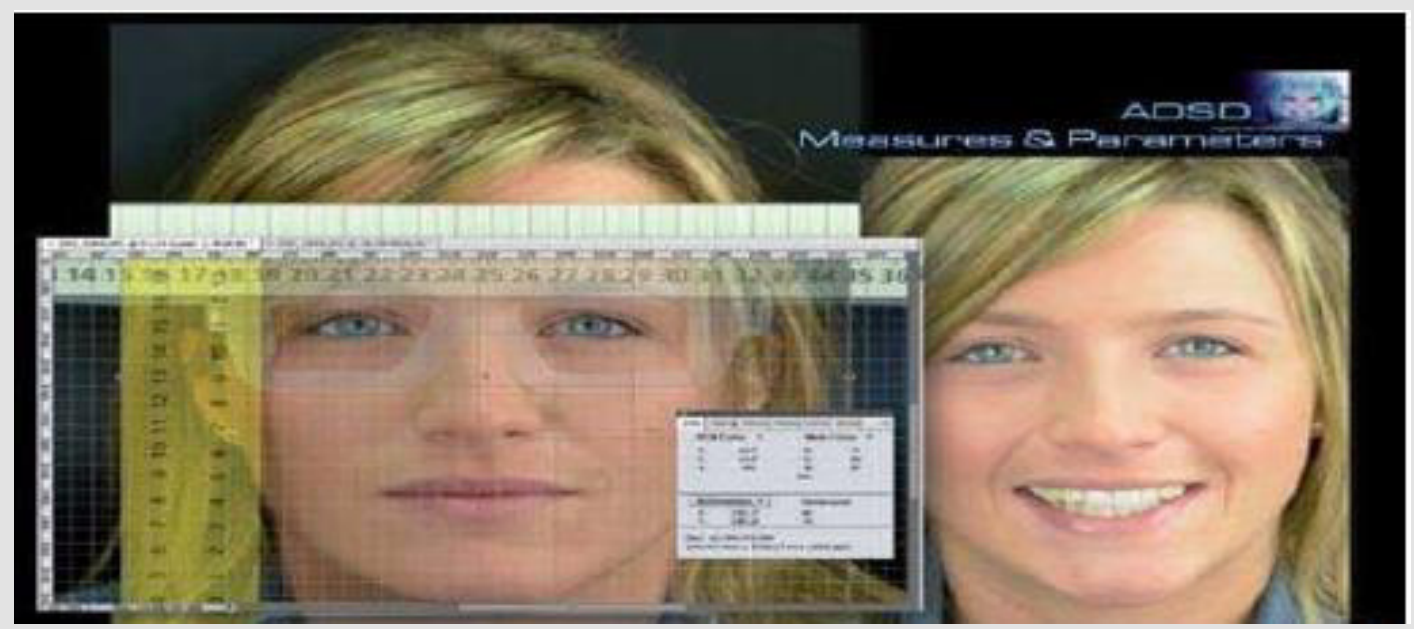

Figure 2: Face Analogic Transport Support. 
If more accurate and detailed measurements of the teeth and gingival parameters are required, one can use digital calipers whose tips are placed at the cervical margin and incisal edge (the length of the tooth) or at the mesial and distal margins relative to the dental line (width of the tooth; Figure 3). These measurements when transmitted can be very effective in communication between the dentist and dental technician, whose manufacturing skills and expertise will be the most important to the end-result of this innovative method. It is necessary to bear in mind that the measurements expressed in millimeters in relation to the digital image produced by the digital processing, as well as the design of the dental contours, are not of much interest to patients, who desire a photograph of the first phase simulation, but the measurements represented as 3-D wax models and mock-ups tried in and analyzed in the patient's mouth will give you an idea of the delicate psychoaesthetic approach to the clinical case very important for aesthetic dentistry [5].

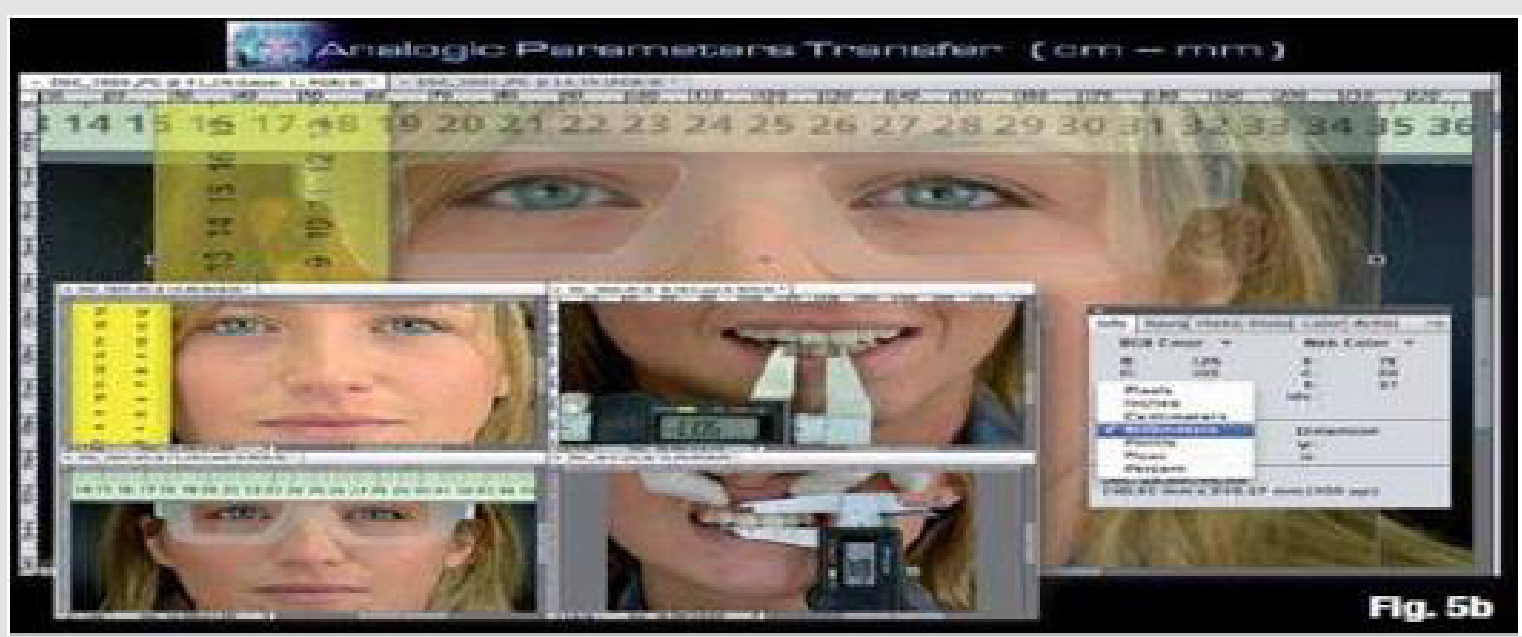

Figure 3: Transfer of analogue measurements to digital calliper.

\section{Analysis Components for Face and a Smile}

In relation to the manner in which to portray the patient in a photograph, we should reflect on the aesthetic component of the face and the smile. For the objective aesthetic analysis, the focal length is modified, starting from the first photograph (Figure 4). For this parameter, the following classification criteria could be applied: a. macro-aesthetics (extra-oral analysis of the face).

b. mini aesthetics (extra-oral analysis of the mouth); and

c. micro-aesthetics (intra-oral analysis of teeth and gingiva).

As regards the aesthetic analysis of the smile, the specific areas of the objective analysis that are pertinent to dentistry are as follows, based on that provided by a number of many authors:

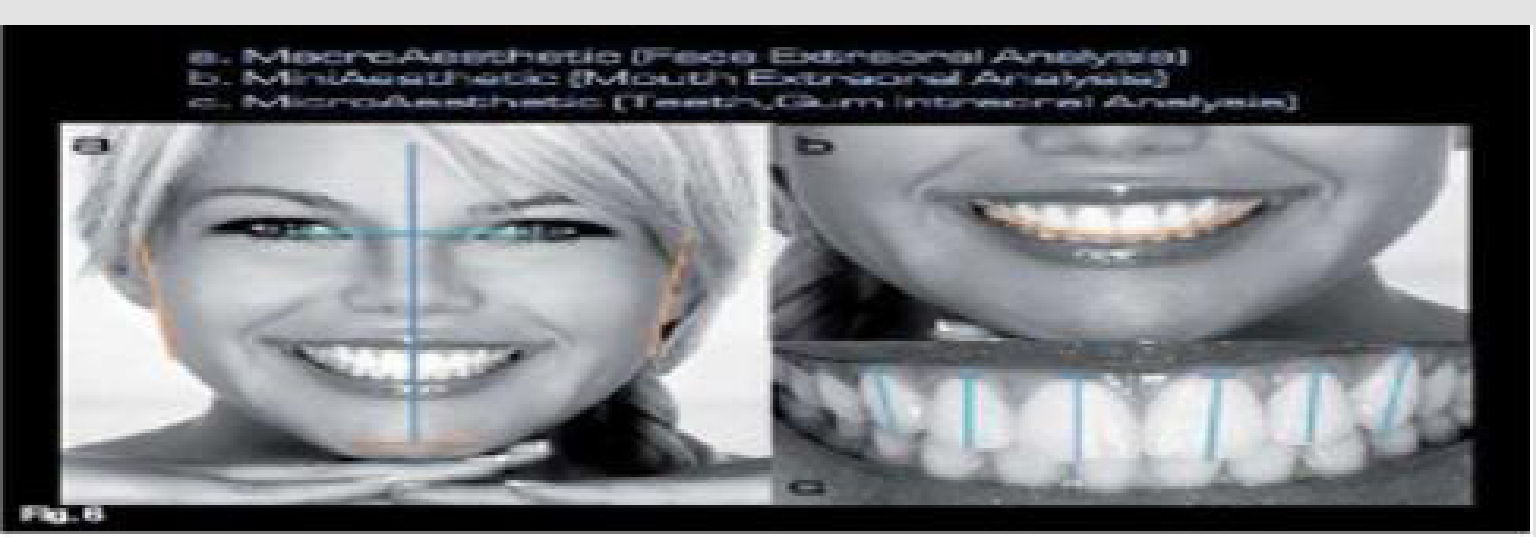

Figure 4: Focal length and analysis of the aesthetic component. 
1. Visagism and Facial analysis: Frontal/lateral, determining morphological features, horizontal/vertical reference lines, vertical/horizontal facial proportions, golden ratio, horizontal/ vertical dimensions, analysis of the facial profile, and analysis of the lips, nose and eyes as regards position and size (Figure 5).

2. Dento-Labial Analysis: Labial dynamics, smile line, width of smile, labial corridors, occlusal plane, midline, and inter- incisal and commissural lines (Figure 6).

3. Gingival Analysis: Architecture, shape parallelism, symmetry, zenith, papillae, biotype and color (Figure 7).

4. Dental analysis: Dental composition, dental arrangement and position, dimensions, proportions, shapes, contours, margins, textures, surfaces, axial inclinations, inter-incisal angles, interproximal contacts and color (Figure 8) [5].

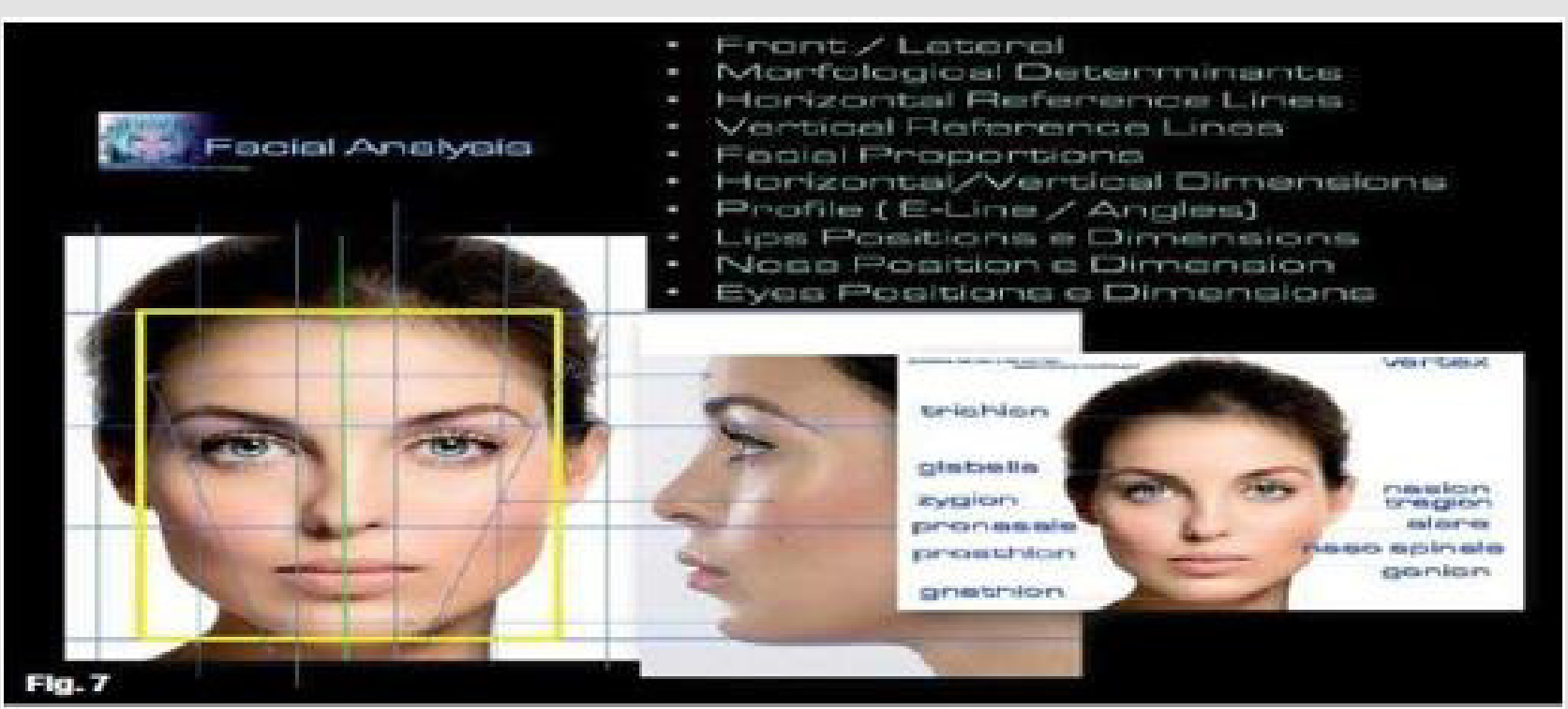

Figure 5: Facial Analysis.

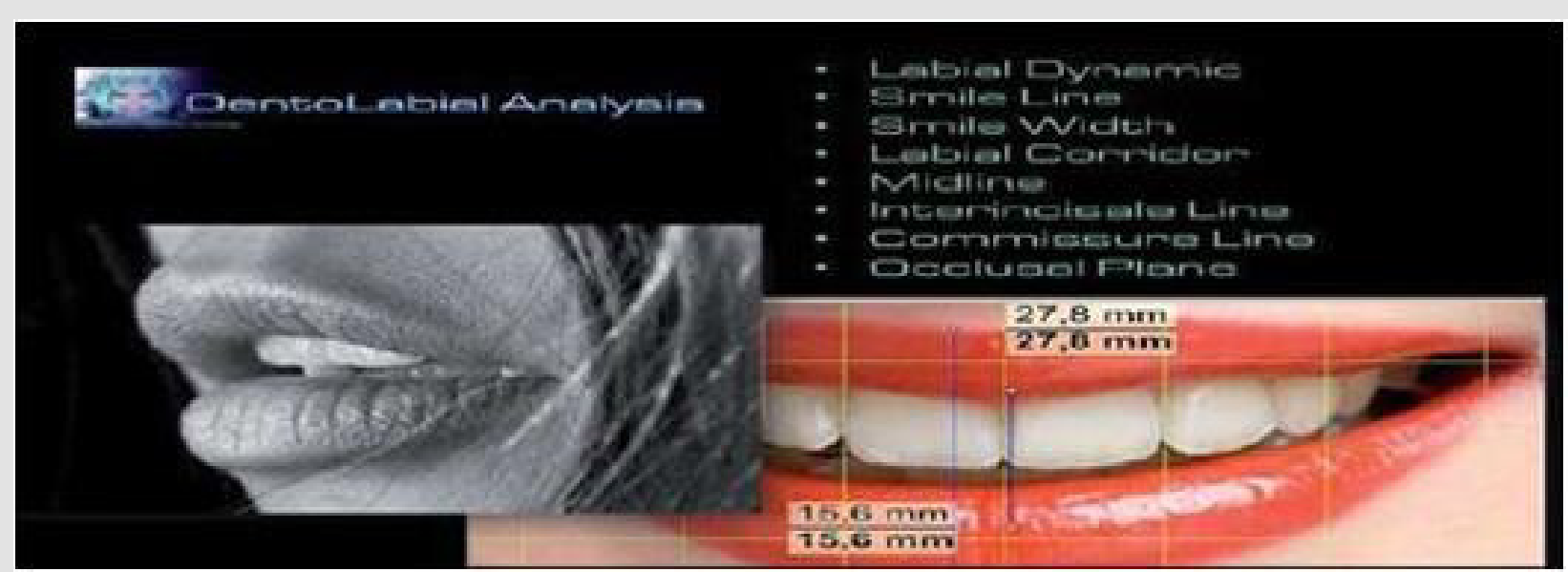

Figure 6: Dento-labial analysis. 


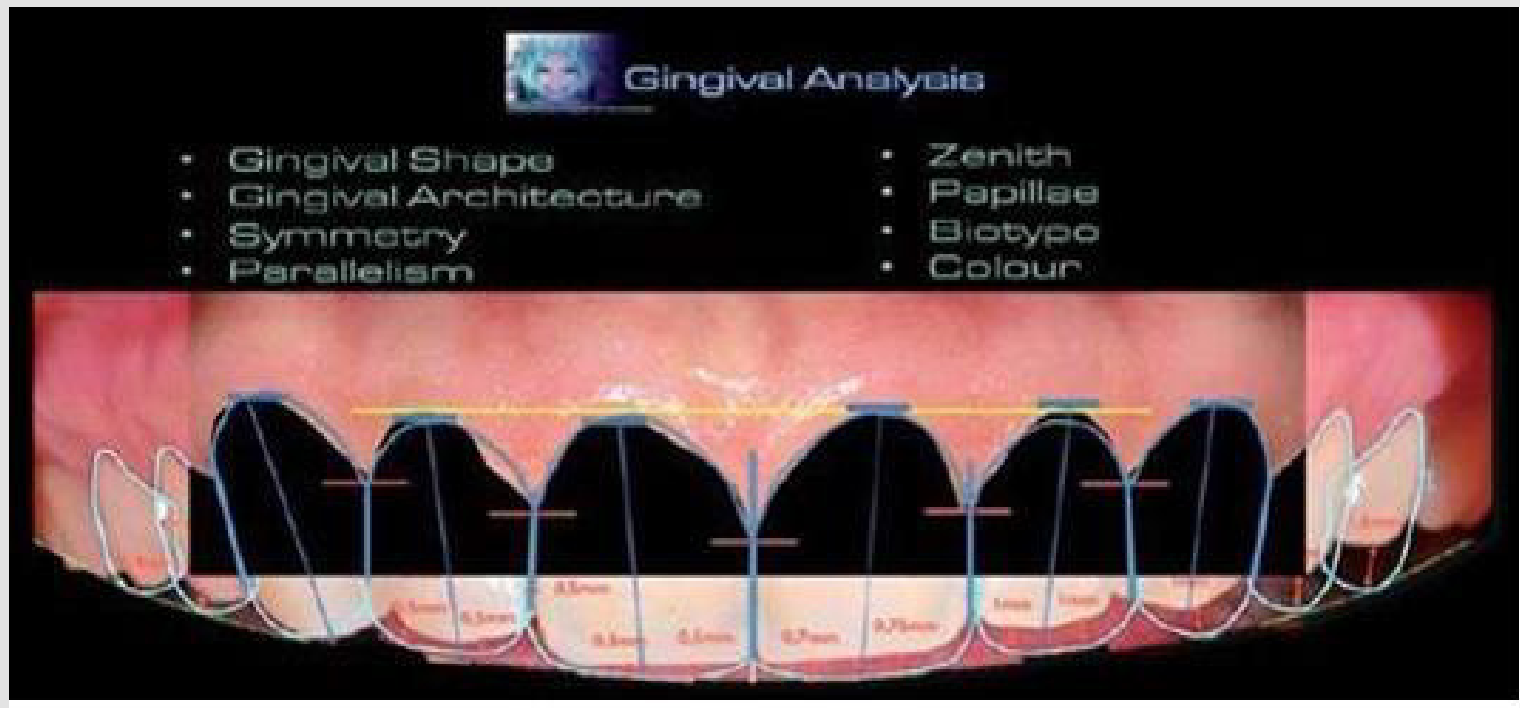

Figure 7: Gingival analysis.

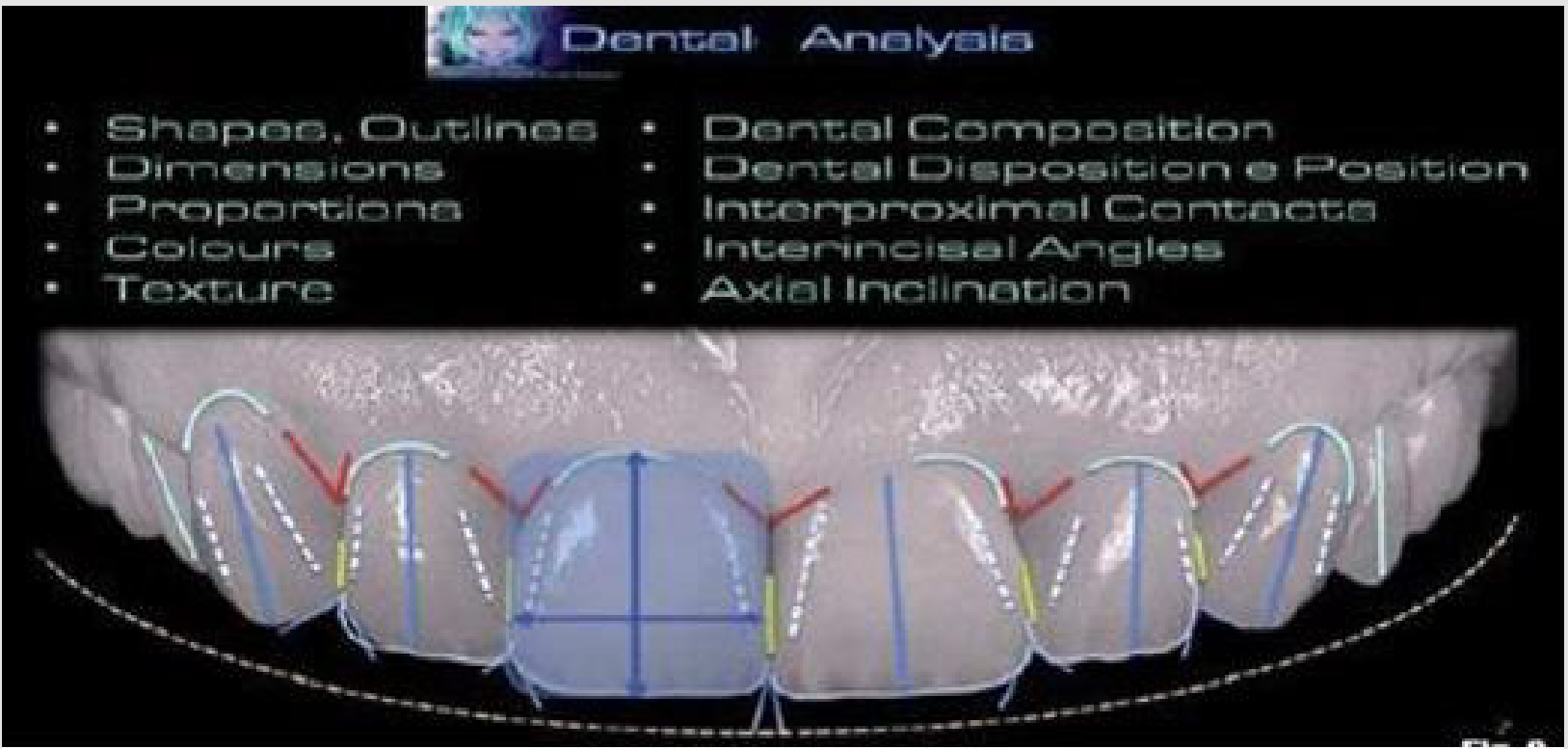

Figure 8: Dental analysis.

\section{Visagism and Facial Analysis}

The final esthetic results may fail to meet the patient's expectations due to disharmony between the smile design and the patient's personality. The patient may feel that the restored teeth do not really "belong" to him or her. For decades, dental clinicians have sought to harmonize the shapes of the teeth with the entire face based on parameters such as gender, personality, and age; however, truly successful results have been elusive.

The visagism concept helps dental clinicians provide restorations that account not only for esthetics, but also for the psychosocial features of the created image, which affect patients' emotions, sense of identity, behavior, and self-esteem.

The shape of the face in relation to the four temperaments can be described as follows [6] (Figure 9):

The first point that should be evaluated by a dentist is the patient's face, because the face has the original structure representing the human characters. In particular, the front face of the analysis affects how the construction of restoration. In this way, we want to take the features into consideration to concentrate on the face and hide abnormal structures. 

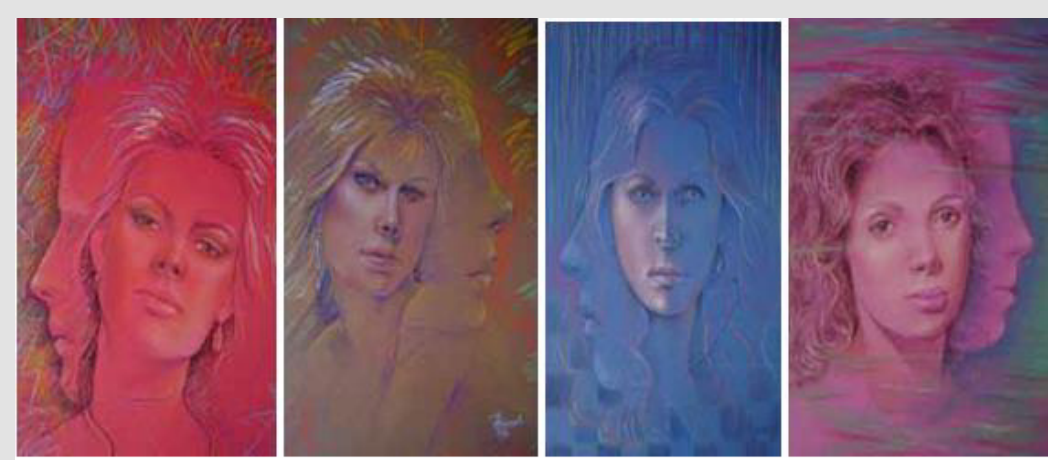

Choleric/strong
temperament:
Determined,
objective,
explosive, intense,
and passionate.

Sanguine/dynamic temperament: extroverted, expansive, joyful, full of life, and enthusiastic.

\section{Melancholic/sensiti ve temperament: Organized, meticulous, perfectionist, timid, reserved, and with a great capacity to think abstractly. \\ Phlegmatic/peaceful temperament: diplomatic, pacifist, mystic, and spiritual, but with a tendency to be apathetic and conformist.}

Figure 9: The shape of the face in relation to the four temperaments.

A. Morphophysiology: Sagittal and frontal axis of the face divided into 3 parts including upper, middle and lower. The upper part is a hairline and glabella; the middle part is a glabella and subnasal; the lower part is subnasal and the area between the soft palate and the menton.

This morphological segmentation represents the specific psychological characteristics to the individuals; upper part of creativity; middle section with emotion; the lower section is characterized by emotional and instinctive activity. These segments should reach the equilibrium and should be in equal size. So as much as possible they should bring each other closer. Middle big trio, a people in order to compensate it can be the teeth longer, so the equilibrium is achieved. The last and the most objective face evaluation methods is geometry-based foundation by assessing the beauty of mathematics. Ideal facial features associated with the golden ratio also do not have much significance in dentistry. But in aesthetic and reconstructive surgery it has an important role in determining the rate of one hundred gold borders. Geometric of the face evaluation is to imagine the lines of the frontal and sagittal angle on the face. These lines are pieces that are brought together to create a horizontal symmetry and facial composition.

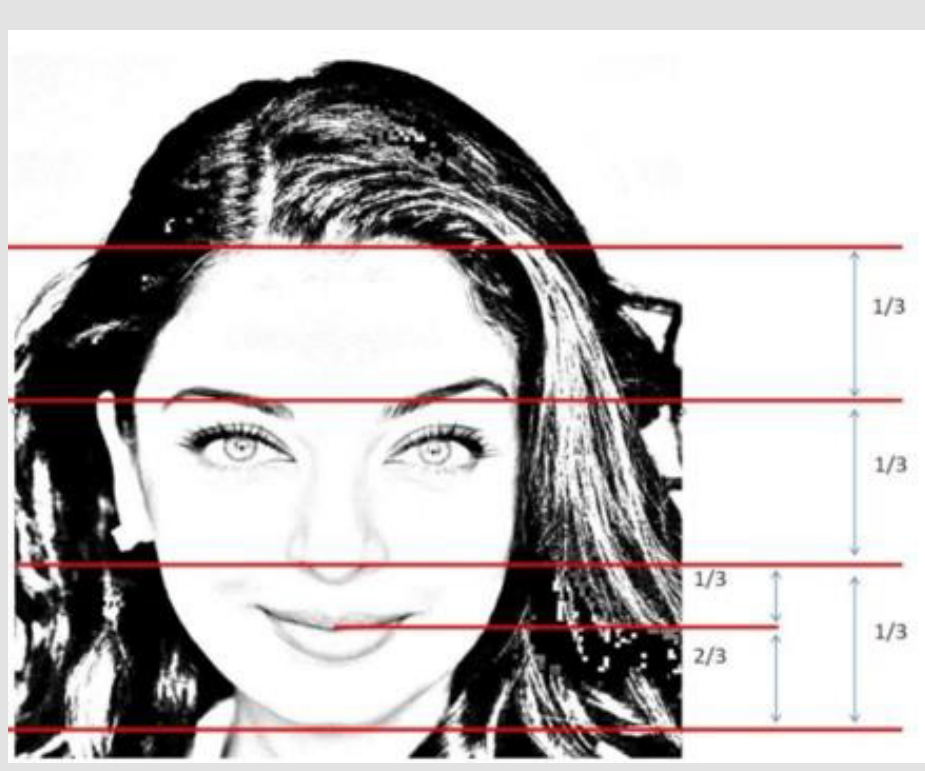

Figure 10: In the triple bottom of the face, the incisal edge with nose base / one-third of the distance between the lip of this area, must cover the remaining two-thirds which is up from the jaw to the incisal edge. 
When nasal frontal perspective, dental and mental midline of face midline (interpupillary perpendicular to the middle distance) will be asked to conflict. Occlusal plane must be perpendicular to the midline. In appealing face is generally applicable rule of thirds, upper, middle and lower face height should be one third of the vertical dimension of the face. In the triple bottom of the face, the incisal edge with nose base / one-third of the distance between the lip of this area, must cover the remaining two-thirds which is up from the jaw to the incisal edge. (Figure 10). In the sagittal angle the nasolabial angle in man must be $90-95^{\circ} \mathrm{C}$, in women must be between $100-105^{\circ} \mathrm{C}$. In examine of E plane should be in 2-4 mm posterior to the line connecting the tip of the chin, the tip of the nose and upper lip (Figure 11) [7].
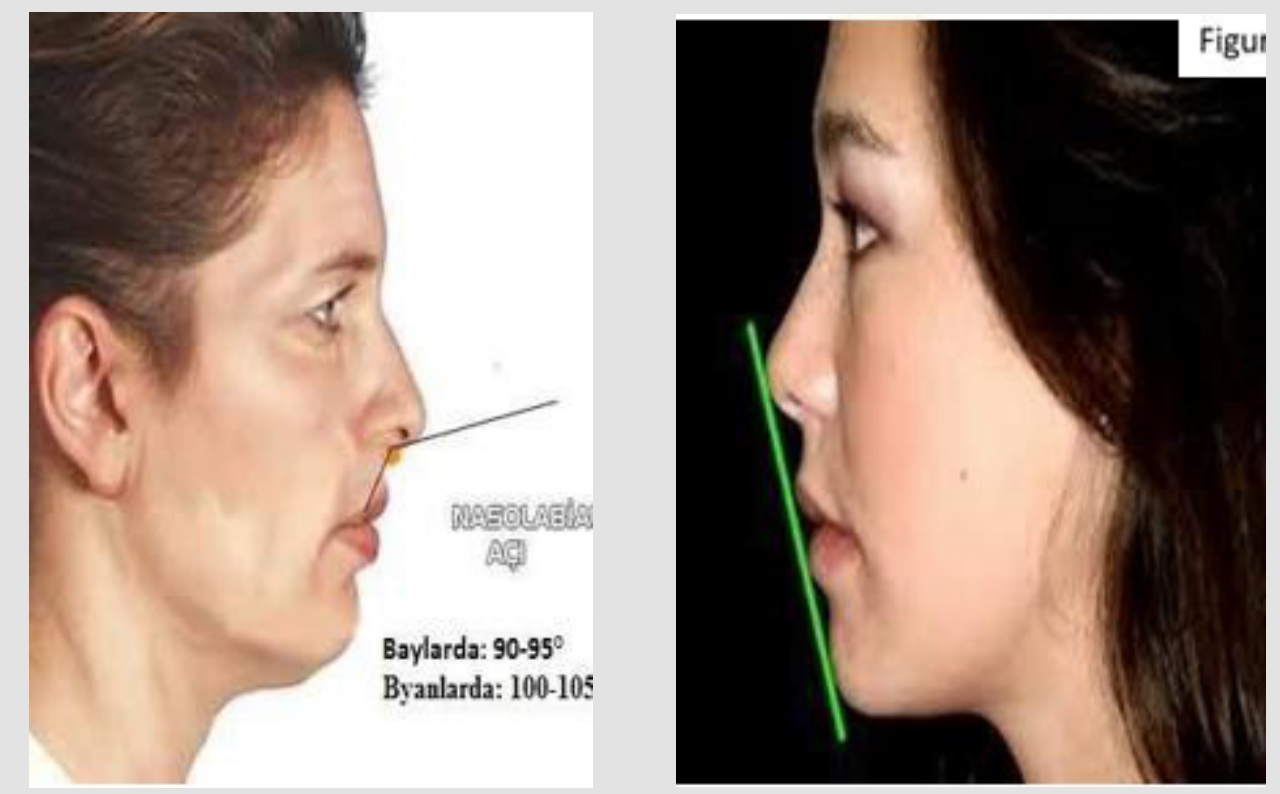

Figure 11: The line connecting the tip of the chin, the tip of the nose and upper lip.

\section{Dentogingival Esthetics Analysis}

Gingival margin placement-and the scalloped shape, in particular-are well discussed. As gingival heights are measured, heights relative to the central, lateral, and canine in an up/down/ up relationship are considered esthetic (Figure 12). However, this may create a false perception that the lateral gingival line is incisal to the central incisor. Rather, in the most esthetic tooth relationships, the gingival line of the four incisors is approximately the same line (Figure 12), with the lateral perhaps being slightly incisal. The gingival line should be relatively parallel to the horizon for the central and the laterals and symmetric on each side of the midline. The gingiva contours (i.e., gingival scallop) should follow a radiating arch similar to the incisal line. The gingival scallop shapes the teeth and should be between 4 to $5 \mathrm{~mm}$ (Figure 13). Related to normal gingival form is midline placement [8-10].

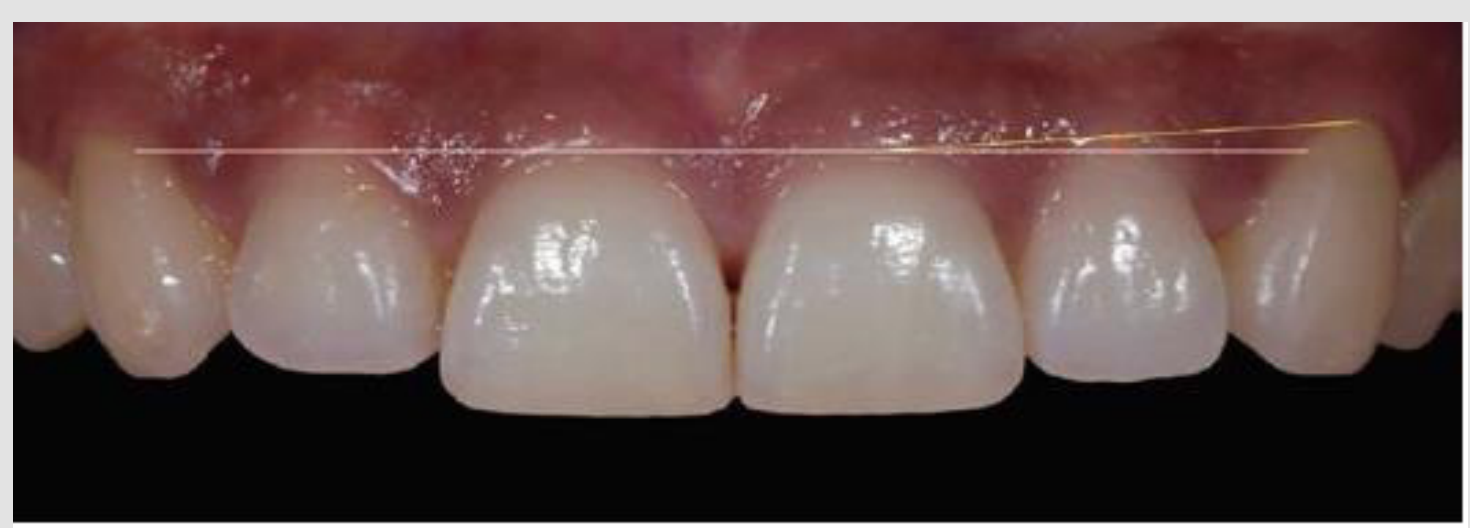

Figure 12: Gingival heights are measured, heights relative to the central, lateral, and canine in an up/down/up relationship are considered esthetic. 


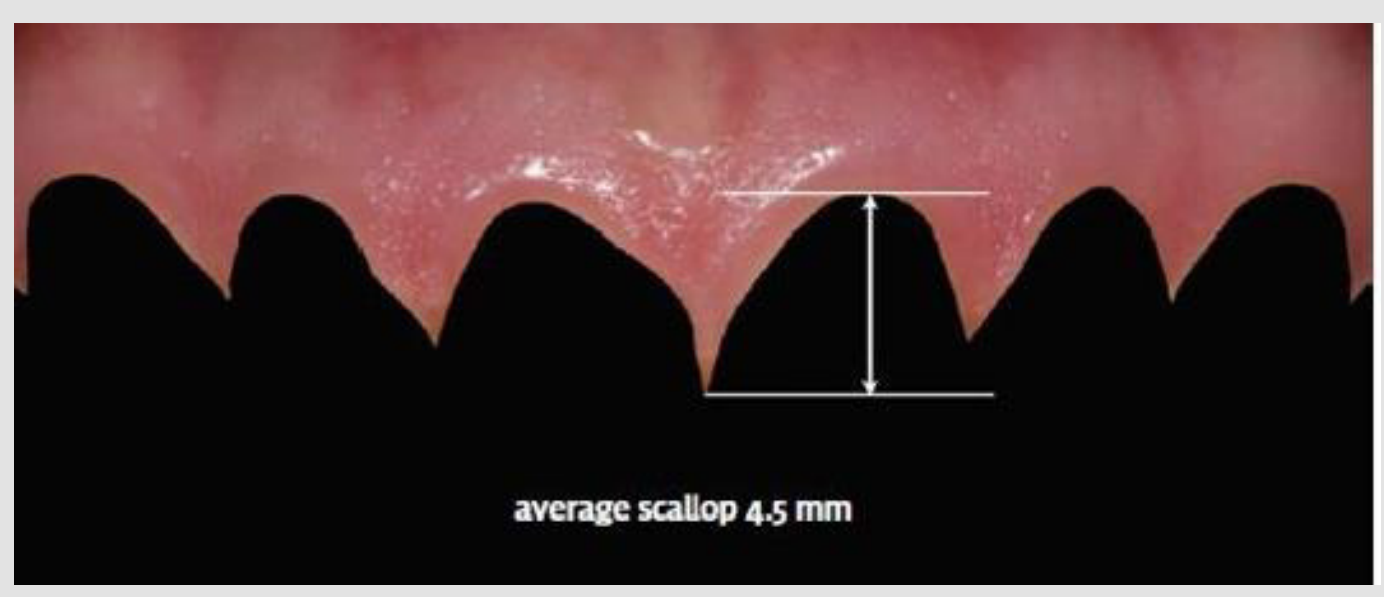

Figure 13: The gingival scallop shapes the teeth and should be between 4 to $5 \mathrm{~mm}$.

Although usually the first issue addressed in smile design, it is not as significant as tooth form, gingival form, tooth shape, or smile line. Several rules can be applied when considering modifying the midline to create an esthetic smile design.

a. The midline only should be moved to establish an esthetic intra-and inter-tooth relationship, with the two central incisors being most important.

b. The midline only should be moved restoratively up to the root of the adjacent tooth.

c. If the midline is within $4 \mathrm{~mm}$ of the center of the face, it will be esthetically pleasing. d. The midline should be vertical when the head is in the postural rest position [3].

\section{Dental Esthetics Analysis}

Part of evaluating dental esthetics for smile design is choosing tooth shapes for patients based on their facial characteristics (e.g., long and dolichocephalic, or squarish and brachiocephalic). When patients present with a longer face, a more rectangular tooth within the esthetic range is appropriate. For someone with a square face, a tooth with an $80 \%$ width to length ratio would be more appropriate, but esthetic smiles could demonstrate ratios between 70 and $75 \%$ or 80 to $85 \%$ (Figure 14) [11-14].
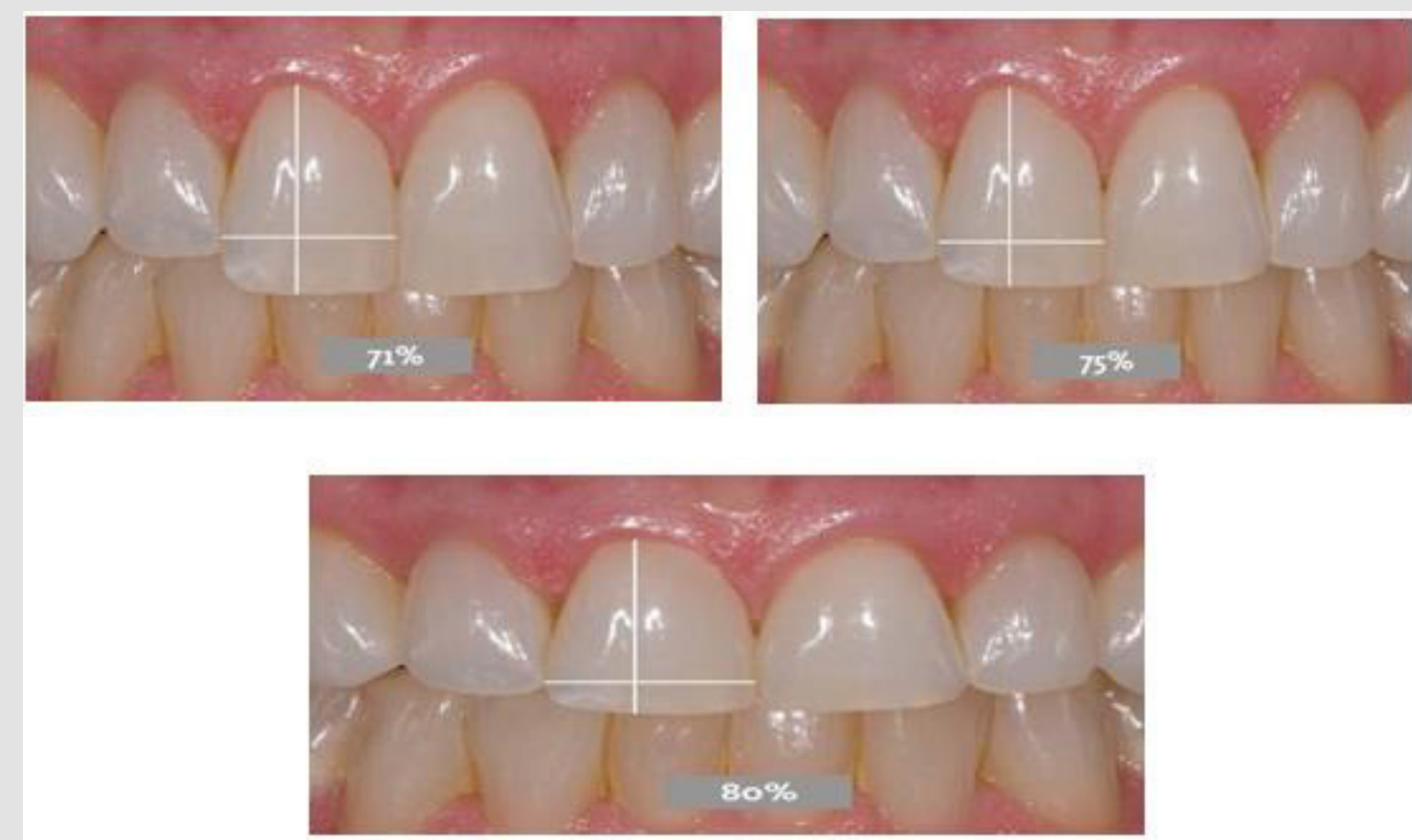

Figure 14: Acceptable width-to-length aspect ratios fall between 70 to $85 \%$, with the ideal range between 80 to $85 \%$. 
The length of teeth also affects esthetics. Maxillary central incisors average between 10 to $11 \mathrm{~mm}$ in length. According to Magne, the average length of an unworn maxillary central to the cemento-enamel junction is slightly over $11 \mathrm{~mm}$. The esthetic zone for central incisor length according to the authors, is between 10.5 and $12 \mathrm{~mm}$, with $11 \mathrm{~mm}$ being a good starting point. Lateral incisors are between $1 \mathrm{~mm}$ to a maximum of $25 \mathrm{~mm}$ shorter than the central, with the canines slightly shorter than the central by between 0.5 to 1 mm (Figure 15). The inter-tooth relationship, or arch form, involves the golden proportion and position of tooth width. Although it is a good beginning, it does not reflect natural tooth proportions. Natural portions demonstrate a lateral incisor between 60 to $70 \%$ of the width of the central incisor, which is larger than the golden proportion. However, a rule guiding proportions is that the canine and all teeth distal should be perceived to occupy less visual space (Figure 16) [15,16].

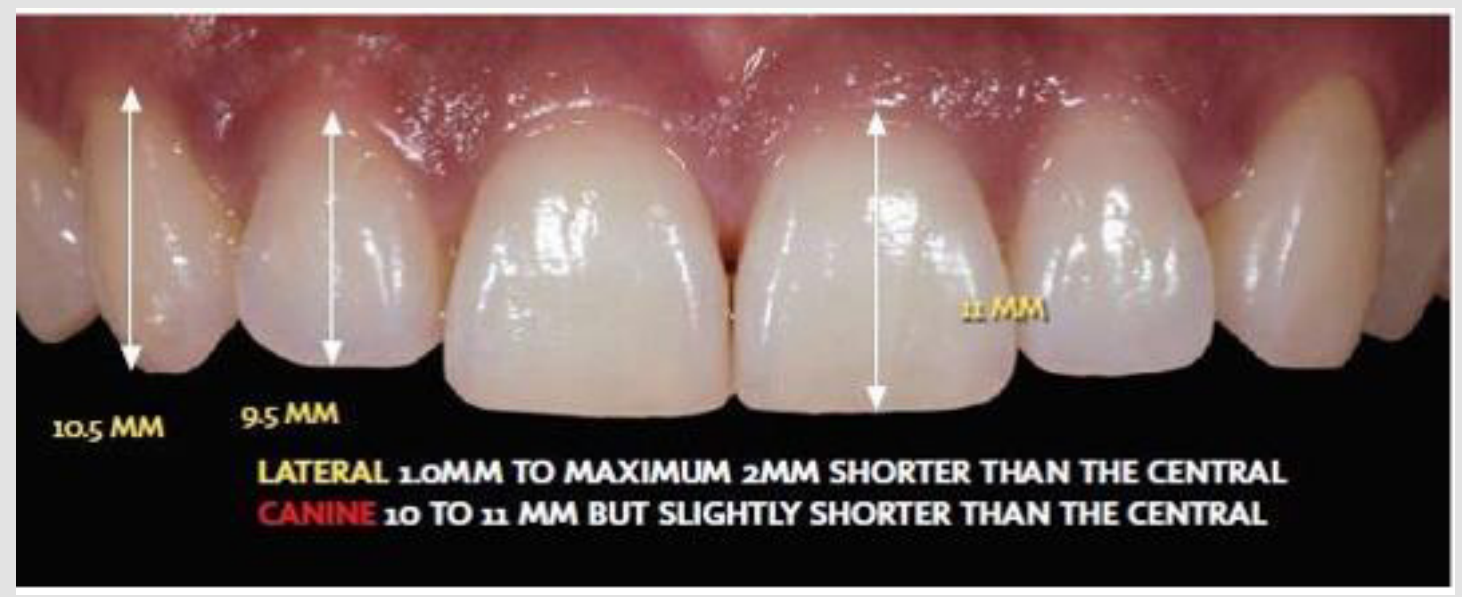

Figure 15: Lateral incisors are between $1 \mathrm{~mm}$ to a maximum of $25 \mathrm{~mm}$ shorter than the central, with the canines slightly shorter than the central by between 0.5 to $1 \mathrm{~mm}$.

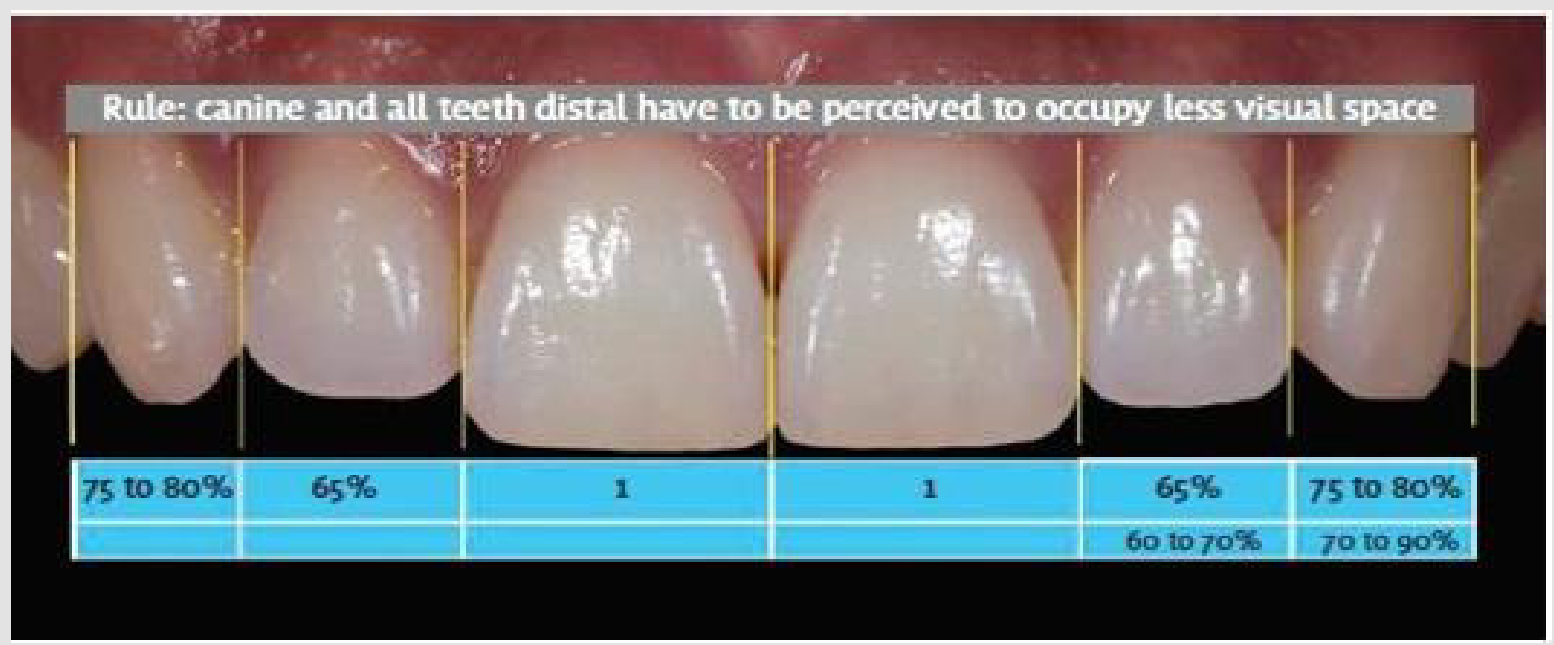

Figure 16: A rule guiding proportions is that the canine and all teeth distal should be perceived to occupy less visual space.

Another rule to help maintain proportions throughout the arch is 1-2-3-4-5; the lateral is $2 / 3$ of the central and the canine is $4 / 5$ of the lateral, with some latitude within those spaces (Figure 17). Finally, contact areas can be moved restoratively up to the root of the adjacent tooth. Beyond that orthodontics is require [3]. The shapes of the anterior teeth are defined by the area that reflects light directly forward, i.e; the area between the cusps of reflection of mesial, distal, cervical, and incisal light, forming the so-called Pincus silhouette. There are five basic tooth shapes: rectangular, triangular, oval, square and trapezoidal (Figures 18), with some possible variations. Vertical, horizontal, inclined, straight, and curved lines interact in infinite ways to create the diversity of natural tooth shapes. 


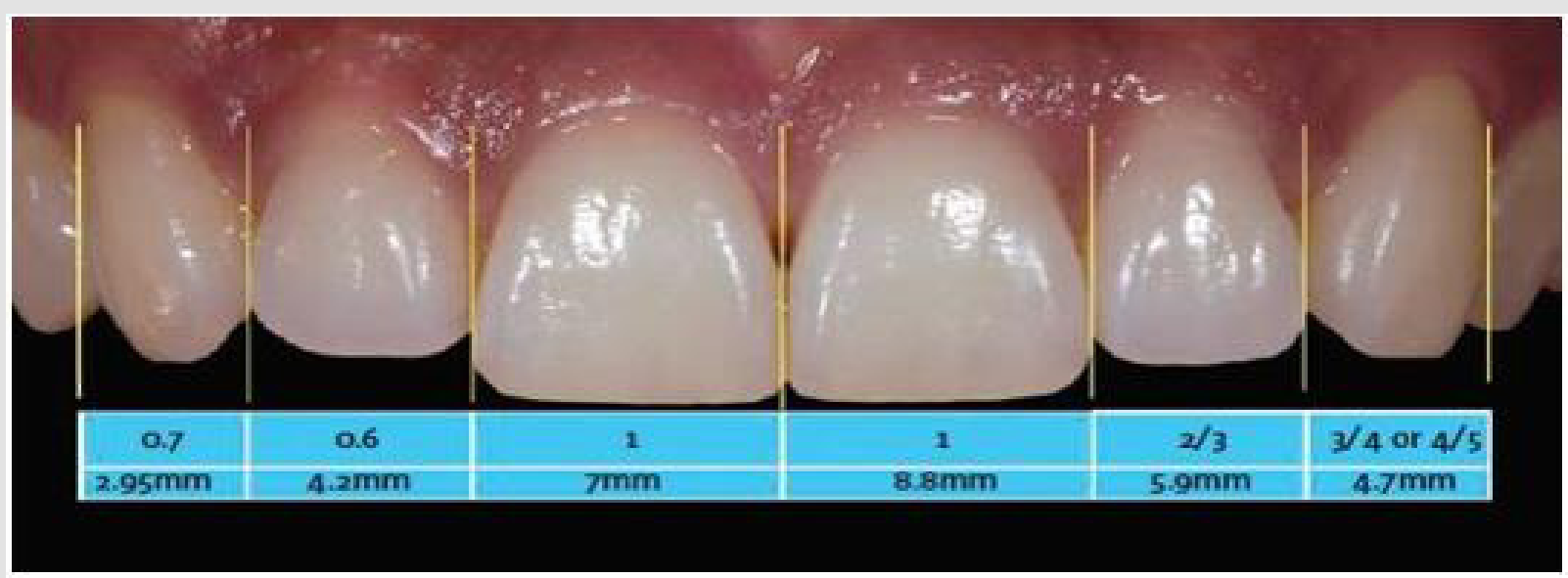

Figure 17: The lateral is $2 / 3$ of the central and the canine is $4 / 5$ of the lateral, with some latitude within those spaces.

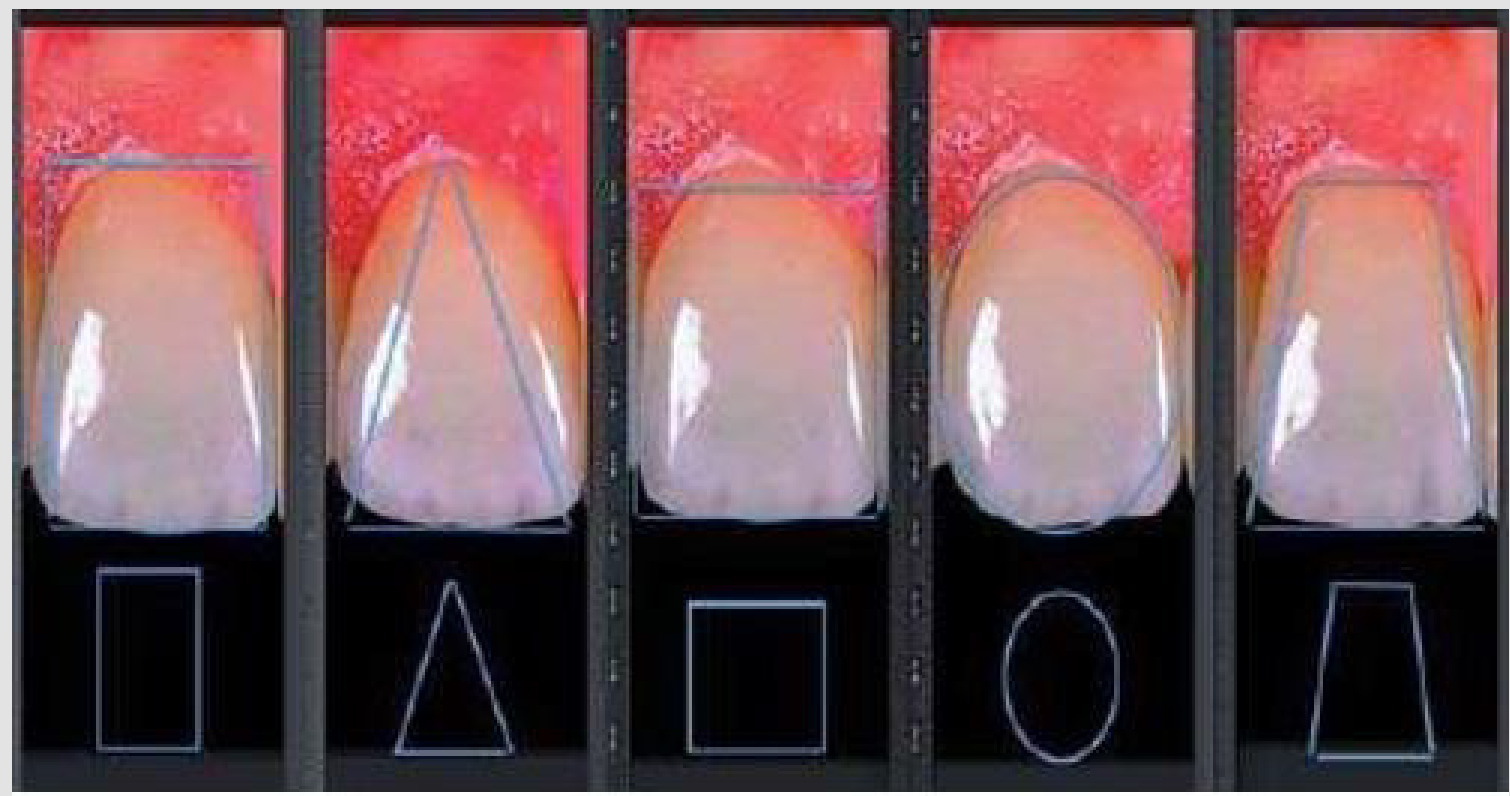

Figure 18: Basic shapes of maxillary central incisors: (a) rectangular, (b) triangular, (c) oval, (d) square, (e) and trapezoidal.

These lines contain their own power of expression and emotional significance, which can be classified as follows:

a. Vertical straight lines represent strength, power, and masculinity.

b. Horizontal straight lines represent the surface on which we are born, live, and die and express equilibrium, passivity, and tranquility. They can also represent a barrier.

c. Inclined straight lines express dynamism, movement, and joy. d. Curved lines represent the gradual transition between two planes (vertical and horizontal) and express gentleness, delicacy, femininity, and sensuality.

The dental arches also follow these basic formats, though it is important to note that variations are frequently encountered (Figure 19). The design of the maxillary anterior teeth, the characteristics of the lips, and the form of the dental arch compose a potent nonverbal message. The esthetic dental design in relation to the four temperaments can be categorized as follows (Figures 20 \& 21): strong, dynamic, sensitive and peaceful [6]. 


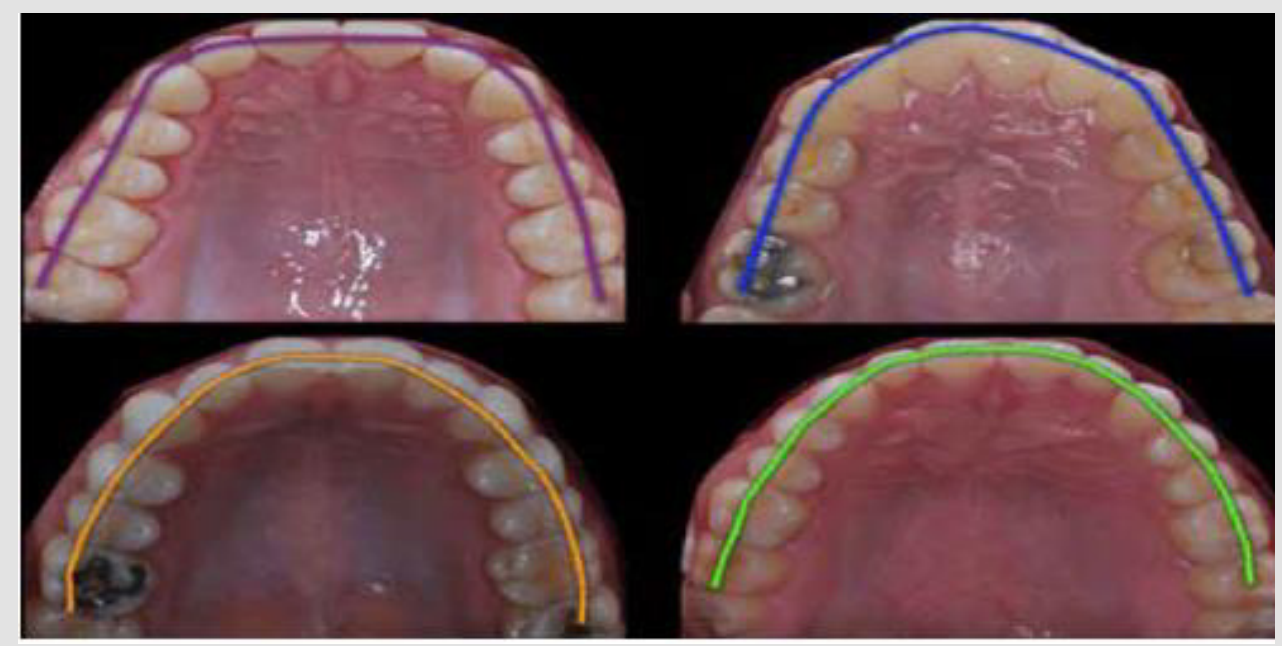

Figure 19: Basic arch forms: (a) rectangular, (b) triangular, (c) oval, and (d) circular.

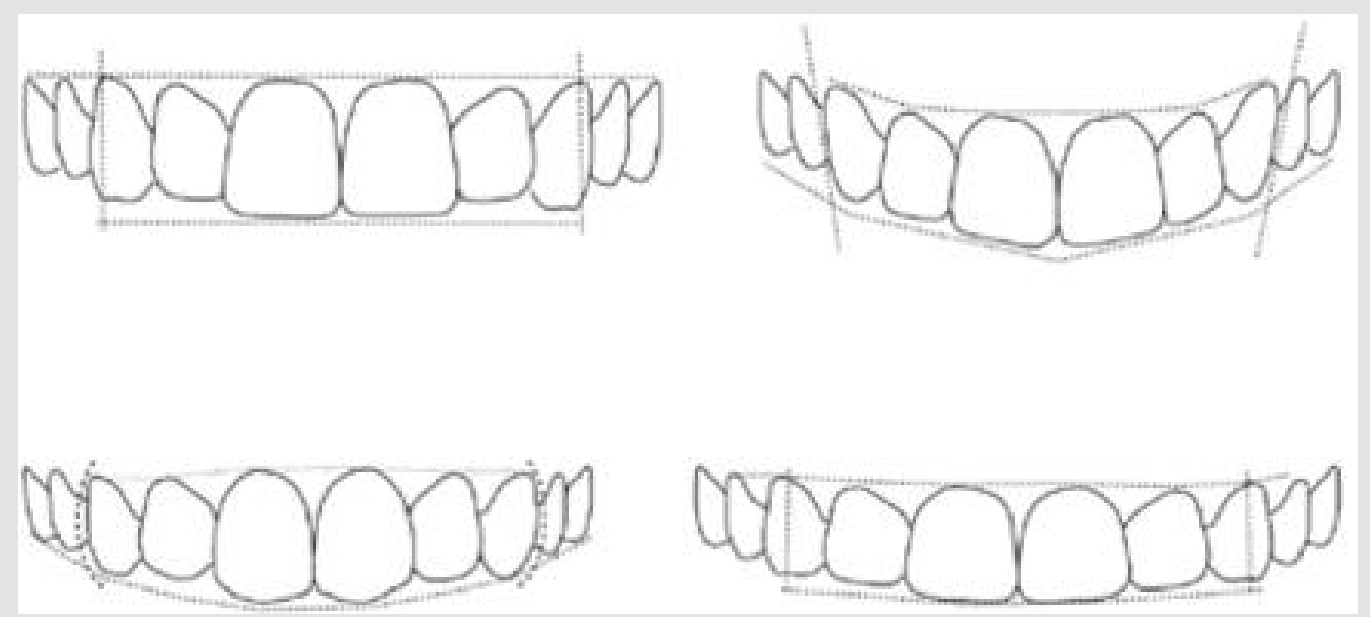

Figure 20: Maxillary arches with esthetic designs in relation to the four temperaments: (a) strong, (b) dynamic, (c) sensitive, and (d) peaceful.

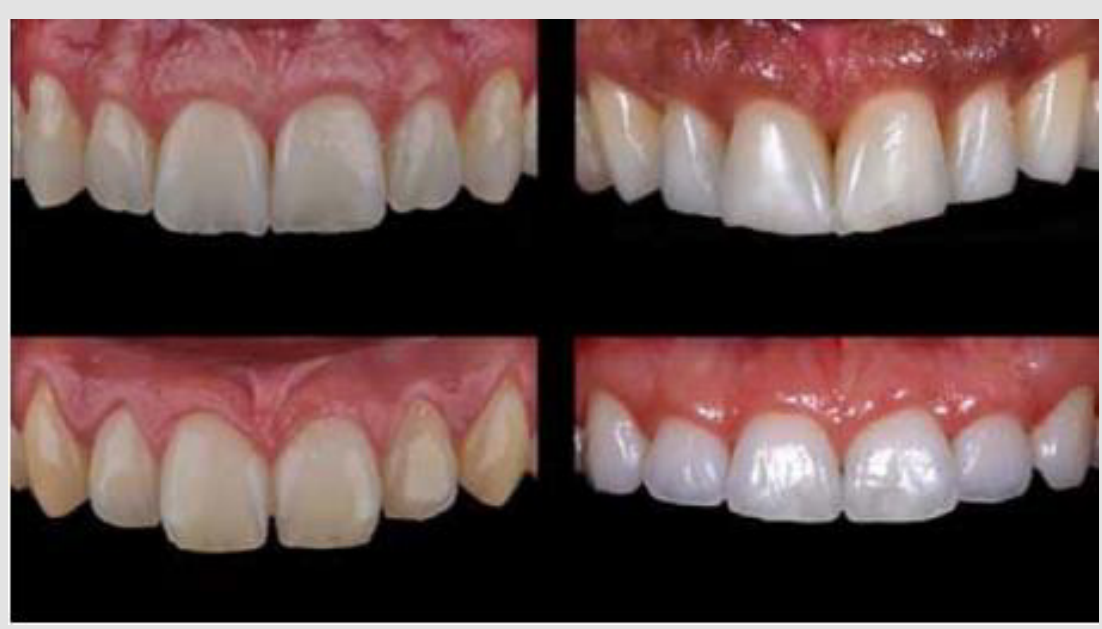

Figure 21: Schematic drawing of maxillary teeth with esthetic designs in relation to the four temperaments: (a) strong, (b) dynamic, (c) sensitive, and (d) peaceful. 


\section{Digital Image Editing and Planning}

Digital image editing can be performed in various ways according to the requirements of the smile designer and with various software packages (both freeware and for purchase) easily obtained from the Web. Their main use includes generic image and photograph editing for both amateur and professional graphic designers. Some of the packages available have been developed by dentists. An important contribution to these packages is offered by some authors, who through the use of Keynote (a presentation application developed by Apple for Mac OS X and iOS) have made smile design easier with results that provide a schematized dental design with real outlines. In addition to Digital Dental Design (Figure 22), DSD offers important processing functions: the import, conversion and editing of dental shapes and types of dentition in the form of real images [5].
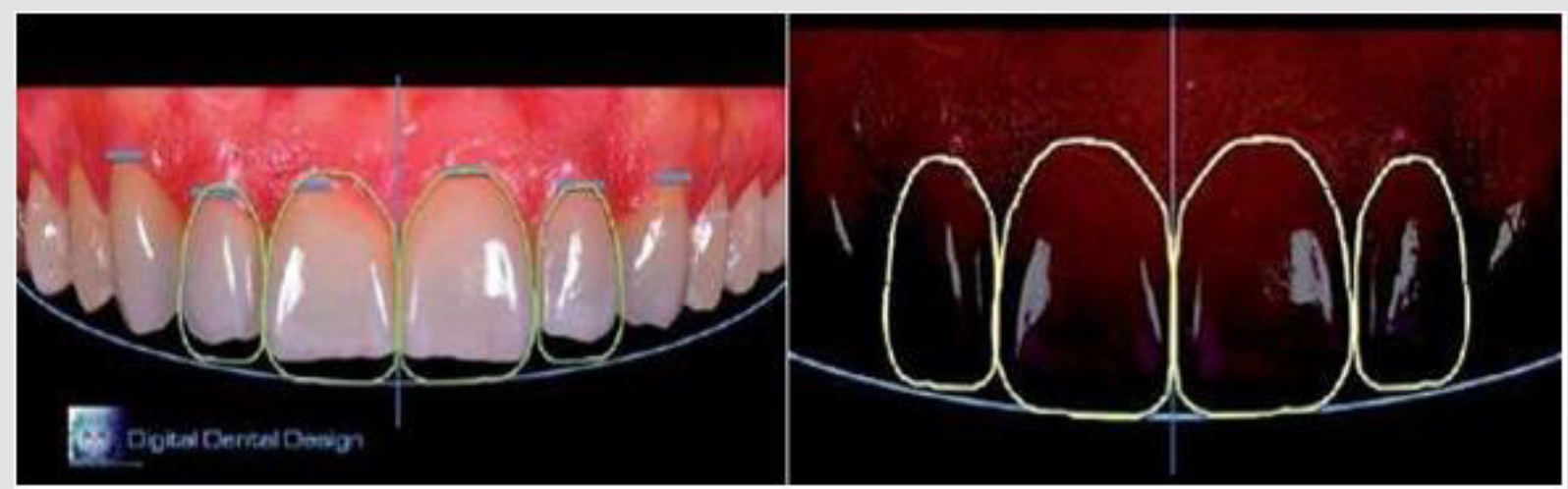

Figure 22: Digital Dental Design, outlines.
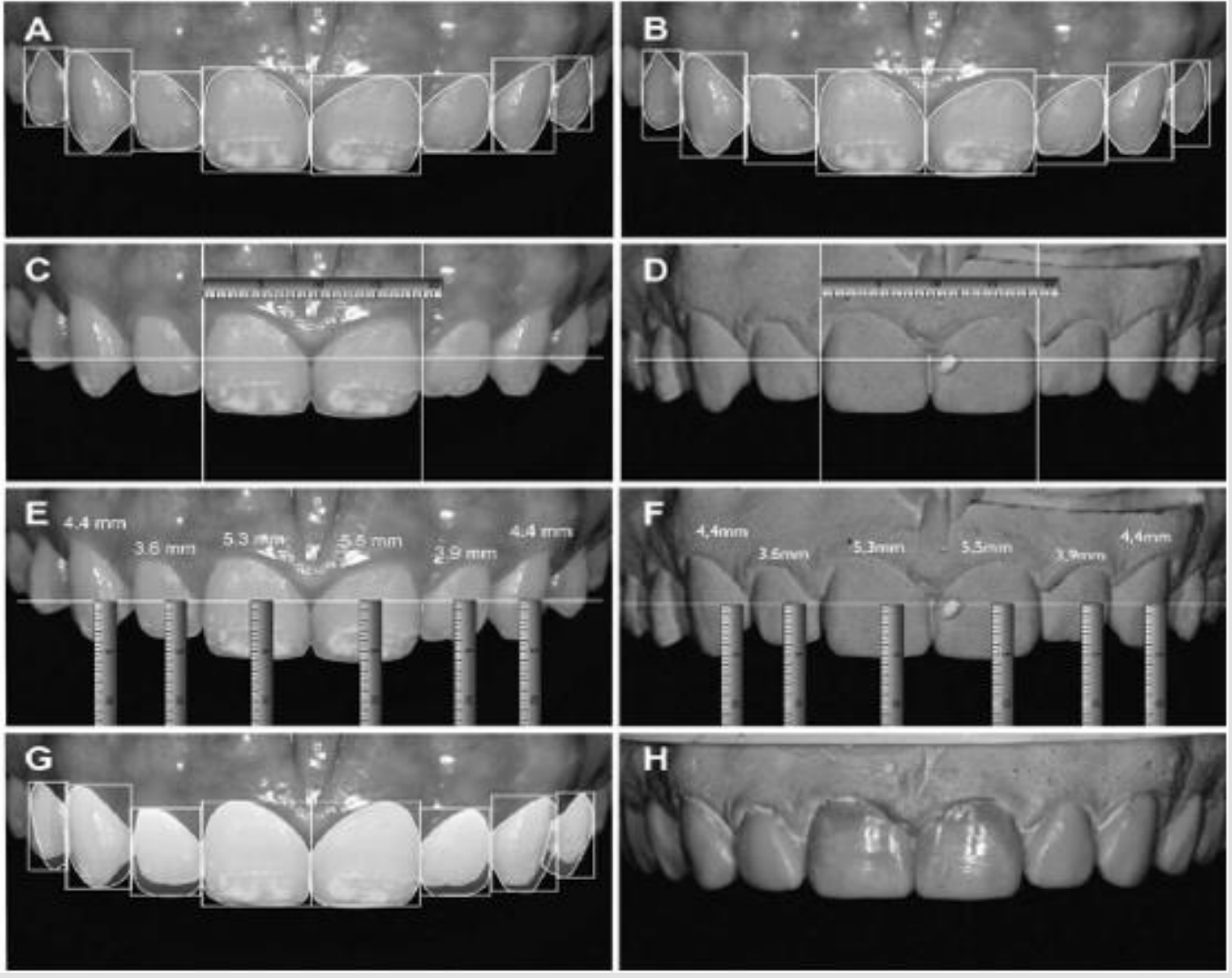

Figure 23: Digital smile design protocol. 
DSD can be used to increase professional/patient communication and to provide greater predictability for the smile's esthetic rehabilitation. Planning through DSD allows an esthetic analysis of the assembly - face, smile, periodontal tissue, and teeththrough the analysis of extra- and intraoral digital photographs, in which the reference lines of the face and of the anatomical axes are plotted as a guide to establish a proper gingival contour, shape, and dental alignment. This provides greater predictability of treatment because it allows a final dental outline showing the relationship between the preoperative situation and the ideal design, in addition to assisting as a guide to diagnostic wax-up and consequently to the mock-up [4]. After the facial analysis the dental analysis was performed. A rectangle with the actual proportion of maxillary teeth and tooth outline were accomplished to analyze the shapes and width/length proportions of the pretreatment teeth (Figure 23) $[17,18]$

Then a rectangle with the ideal length/width proportion was placed over the teeth to compare the actual pretreatment proportions with the ideal ones (Figure 23B). Furthermore, measurements in the digital photographs were performed and transferred to the dental cast to calibrate the digital ruler and guide the diagnostic wax-up (Figure 23C-F). The final teeth outline, and gingival contour were planned (Figure 23G), and the diagnostic wax-up was fabricated using DSD as a guide (Figure 23H) [5].

\section{Result}

The DSD is a tool that assists with the diagnostics and allows the clinician to better predict treatment outcomes by using analysis of the esthetic principles in extra- and intraoral digital photographs [4]. Knowledge of smile design, coupled with new and innovative dental technologies, allows dentists to diagnose, plan, create, and deliver esthetically pleasing new smiles. Simultaneously, digital dentistry is enabling dentists to provide what patients demand: quick, comfortable, and predictable dental restorations that satisfy their esthetic needs [1]. In addition, implementation of a digital dentistry tool can improve communication among the patient, clinician, and dental laboratories and may become a common technique for all esthetic rehabilitations. The DSD is a simple technique that does not require specific equipment or software [4].

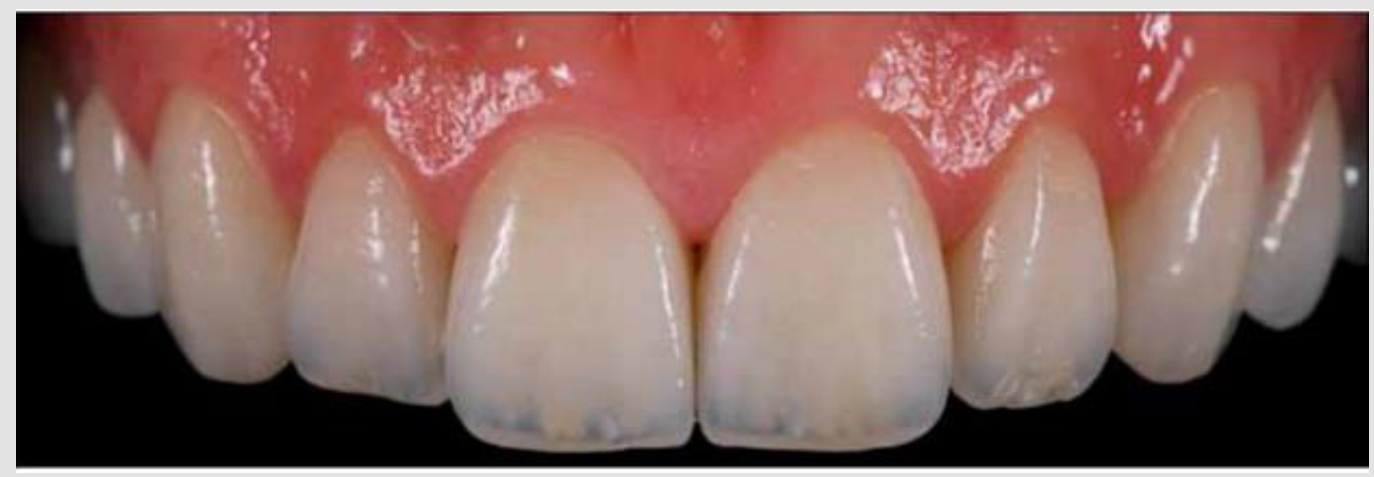

Figure 24: Analysis of the esthetic principles in extra- and intraoral digital photographs.

\section{References}

1. Bhuvaneswaran M (2010) Principles of smile design. Journal of Conservative Dentistry 13(4): 225-232.

2. Ward DH (2015) Proportional Smile Design. Dental Clinics of North America 59(3): 623-638

3. Mclaren EA, Culp L (2013) Smile analysis - The Photoshop smile design technique: Part I. Journal of Cosmetic Dentistry 29(1): 94-108.

4. Meereis C, de Souza G, Albino L, Ogliari F, Piva E, et al. (2015) Digital smile design for computer-assisted esthetic rehabilitation: Two-year Follow-up. Operative Dentistry 41(1): E13-E22.

5. Bini V (2015) Aesthetic digital smile design: Software-aided aesthetic dentistry- Part II. CAD/CAM International Magazine of Digital Dentistry 5(2): 12-17.

6. Paolucci B, Calamita M, Coachman C, Gurel G, Shayder A, et al. (2012) The Art of dental composition. Quintessence of Dental Technology pp. 187-201.

7. Yasmin Kulak Ozkan, Burçin Akoglu Ozkan (2014) Smile design in aesthetic restorations from the book. (Estetik restorasyonlarda gülüşdizaynı kitaptan).
8. Saiesha Mistry (2012) Principles of smile demystified design. Journal of Cosmetic Dentistry 28(2): 116-124

9. Nalla Sandeep, Parth Satwalekar, Siva Srinivas, Chandra Sekhar Reddy, G Ramaswamy Reddy, et al. (2015) An Analysis of maxillary anterior teeth dimensions for the existence of golden proportion: Clinical study. Journal of International Oral Health 7(9): 18-21.

10. Sambhav Jain, Munish Reddy, Pradeep Raghav, Shalu Jain, Arbab Anjum, et al. (2015) Assessment of tooth proportions in an aesthetically acceptable smile. Journal of Clinical and Diagnostic Research 9(4): 7-10.

11. Peter M Prendergast (2012) Facial proportion. Advanced surgical facial rejuvenation pp. $15-22$.

12. Schabel BJ, Baccetti T, Franchi L, McNamara JA (2010) Clinical photography vs digital video clips for the assessment of smile esthetics. The Angle Orthodontist 80(4): 490-496.

13. McLaren EA, Garber DA, Figueira J (2013) The Photoshop Smile Design technique (part 1): digital dental photography. Compend Contin Educ Dent 34(10): 772-776.

14. Design ES (2014) Esthetic Smile Design. Journal of Cosmetic Dentistry 29(4): 102-117. 
15. Ackerman MB, Ackerman JL (2002) Smile analysis and design in the digital era. Journal of Clinical Orthodontics 36(4): 221-236.

16. Thumati PSP, Manwani R, Mahantshetty M (2014) Topic - Digital Smile Designing (Dsd) Protocol to Rehabilitate a Trauma Case- a Case Report Asian Journal of Science and Technology 5(3): 255-259.

\section{ISSN: 2574-1241}

DOI: 10.26717/BJSTR.2020.31.005099

Ammar Kayssoun. Biomed J Sci \& Tech Res

(C) This work is licensed under Creative

Submission Link: https://biomedres.us/submit-manuscript.php
17. Calamia JR, Wolff MS (2015) The Components of Smile Design: New York University Smile Evaluation Form Revisited, Update 2015. Dental Clinics of North America 59(3): 529-546.

18. Patnaik VVG, Rajan S, Sanju B (2003) Anatomy of "A Beautiful Face \& Smile." J Anat Soc India 52(1): 74-80.

$\begin{array}{ll}\text { BIOMEDICAL } & \text { Assets of Publishing with us } \\ \text { RESEARCHES } & \text { - Global archiving of articles } \\ & \text { - Immediate, unrestricted online access } \\ & \text { - Rigorous Peer Review Process } \\ & \text { - Anthors Retain Copyrights }\end{array}$

